\title{
Symmetry of the Physical Probability Function Implies Modularity of the Lattice of Decision Effects*
}

\author{
Günter Dähn \\ Mathematisches Institut der Universität Tübingen
}

Received April 18, 1972

\begin{abstract}
This paper states two equivalent conditions from which modularity of the lattice $G$ of decision effects $E$ can be derived. It may be seen as a supplement to Ludwig's approach [5] to an axiomatic foundation of physical theories. As a consequence of these conditions every filter $\boldsymbol{T}_{E}$ is a self-adjoint projector on the Hilbert space $B^{\prime}$ generated by the decision effects.
\end{abstract}

\section{The Construction of a Canonical Linear Order Isomorphism}

The mathematical symbols and definitions used in the sequel are taken from $[1,2]$ without further explications. Theorem 4 in [1] states the existence of a bijection $\underline{J}$ between the set of all atoms $P$ of $G$ and the set $A(W)$ of all atoms $K_{1}(P)=\left\{V_{P}\right\}$ of $W$. This first part of our paper is concerned with the possibility of extending that bijection $\underline{J}$ to the whole of $B^{\prime}$ such that its extension $J$

(i) becomes a canonical linear isomorphism between $B^{\prime}$ and $B$ and

(ii) preserves order in both directions.

Remark 1. Let us remember a well-known fact from linear algebra: Given two $R$-vector spaces $B_{1}, B_{2}$ and any linearly independent set $S \subset B_{1}, S \neq \emptyset$ :

(a) if $\underline{h}: S \rightarrow B_{2}$ is a map, then there exists a unique linear extension $h: \operatorname{lin}_{\mathbf{R}} S \rightarrow B_{2}$

(b) the linear extension $h$ of $\underline{h}$ is injective iff

$\left(b_{1}\right) \underline{h}$ is injective and

$\left(\mathrm{b}_{2}\right) \underline{h}[S]$ is linearly independent.

Of course, $B^{\prime}, B$ having the same finite dimension, say $N$, they are isomorphic and the isomorphism depends, in general, on the basis chosen. The very problem here is to point out a canonical isomorphism $J$ between $B^{\prime}$ and $B$ such that

$$
J \mid A(G)=\underline{J} \text { and } J^{-1} \mid A(W)=\underline{J}^{-1} .
$$

* This paper extends a final report presented to and supported by the Deutsche Forschungsgemeinschaft. 
Indeed, these two restrictions will guarantee that $J$ is canonical and an order preserving isomorphism. Since $A(G)$ generates $B^{\prime}$, every maximal linearly independent subset $S$ of $A(G)$ is a basis of $B^{\prime}$. Applying (a) and (b) to such an $S$, we have, by hypothesis, $\operatorname{lin}_{R} S=B^{\prime}$ and $J$ injective. Moreover, by $\operatorname{dim} B^{\prime}=\operatorname{dim} B=N$ the unique linear extension $J$ of $\underline{J}$ is even bijective provided the linear independence of $\underline{J}[S]$ could be verified.

To give a motivation though incomplete we define for every $E$ $\in G: \mathscr{S}(E):=\{S(E) \mid S(E) \subset A(G)$ and $S(E)$ generates a covering chain of $E\}$. Then the linear independence of every $S(E)$ can be proved:

Theorem 1. For all $E \in G$ and every $S(E) \in \mathscr{S}(E)$ there holds linear independence of $S(E)$.

Proof. Since $B^{\prime}$ is finite-dimensional, all covering chains of any $E \in G$ are finite. For any $E \in G$ let $S_{r}(E)=\left\{P_{1}, \ldots, P_{r}\right\}$ generate the covering chain $P_{1} \lessdot P_{1} \vee P_{2} \lessdot \cdots \lessdot \bigvee_{i \in \boldsymbol{N}_{r}} P_{i}=E$ of $E$. Without loss of generality, assume, for the non-trivial case $r>1$, the linear dependence of $S_{r}$ such that $P_{r}=\sum_{i \in \boldsymbol{N}_{r-1}} \beta_{i} P_{i}$. Since $\bigvee_{i \in \boldsymbol{N}_{r-1}} P_{i} \neq \mathbf{1}$, so $K_{0}\left(\underset{i \in \boldsymbol{N}_{r-1}}{\bigvee} P_{i}\right)$ $=\bigcap_{i \in N_{r-1}} K_{0}\left(P_{i}\right) \neq \varnothing$ and, because of $P_{r} \pm \bigvee_{i \in N_{r}-1} P_{i}$, there holds $K_{0}\left(\bigvee_{i \in \boldsymbol{N}_{r-1}}^{i \in \boldsymbol{N}_{r-1}} P_{i}\right) \nsubseteq K_{0}\left(P_{r}\right)$. Hence there exists $\left.V^{\prime} \in K_{0}^{i \in \boldsymbol{N}_{r}-1} \bigvee_{i \in \boldsymbol{N}_{r-1}} P_{i}\right)$ such that $\left\langle V^{\prime}, P_{r}\right\rangle \neq 0$. The assumption, however, implies the contradiction $\left\langle V^{\prime}, P_{r}\right\rangle=\sum_{i \in N_{r-1}} \beta_{i}\left\langle V^{\prime}, P_{i}\right\rangle=0$.

Theorem 2. The image $\underline{J}\left[S_{r}(E)\right]=\left\{V_{P_{i}} \mid i \in N_{r}\right\}$ generates a covering chain of $K_{1}\left(\bigvee_{i \in N_{r}} P_{i}\right)$ in $W$ and is linearly independent.

Proof. $G$ and $W$ being isomorphic lattices, there holds $K_{1}\left(P_{1}\right)$ $\lessdot K_{1}\left(P_{1} \vee P_{2}\right) \lessdot \cdots \lessdot K_{1}\left(\bigvee_{i \in N_{r}} P_{i}\right)$ which proves the first assertion. To verify the second one, assume, for the non-trivial case $r>1$, without loss of generality that $\underline{J}\left[S_{r}(E)\right]$ is linearly dependent such that $V_{P_{r}}=\sum_{j \in N_{r-1}} \beta_{j}^{\prime} V_{P_{j}}$. Since $P_{r} \leftrightarrows \bigvee_{i \in N_{r-1}} P_{i}$, so $\left\{V_{P_{r}}\right\}=K_{1}\left(P_{r}\right) \nsubseteq K_{1}\left(\bigvee_{\iota \in N_{r-1}} P_{l}\right)$ $=K_{0}\left(\bigwedge_{i \in N_{r-1}}^{j \in N_{r}-1} P_{i}^{\perp}\right)$, hence $\left\langle V_{P_{r}}, \bigwedge_{i \in N_{r-1}}^{i \in N_{r}-1} P_{i}^{\perp}\right\rangle \neq 0$. Since $\left\langle V_{P_{J}}, \bigwedge_{i \in N_{r}-1} P_{i}\right\rangle^{-1}=0$ for every $j \in N_{r-1}$, the assumption implies the contradiction

$$
\left\langle V_{P_{r}}, \bigwedge_{i \in \boldsymbol{N}_{r-1}} P_{i}^{\perp}\right\rangle=\sum_{j \in \boldsymbol{N}_{r-1}} \beta_{j}^{\prime}\left\langle V_{P_{j}}, \bigwedge_{i \in \boldsymbol{N}_{r-1}} P_{i}^{\perp}\right\rangle=0 .
$$

From Remark 1 together with these theorems there results the 
Corollary. $\underline{J}$ has a linear injective extension $J: \operatorname{lin}_{\mathbf{R}} S_{r}(E) \rightarrow B$.

Remark 2. The proofs make clear that analogous statements can be obtained by starting from $\underline{J}\left[S_{r}\right]$ as a set generating a covering chain in $W$ and then applying $\underline{J}^{-1}$. Hence $J$ preserves linear independence of atoms of covering chains in both directions.

We failed to prove similar properties of $\underline{J}$ for any linearly independent set of atoms in $G$ and $W$, respectively, without subsequently introducing an additional axiom suggested by Ludwig during a seminar at the university of Marburg on foundations of physical theories. This axiom turns out to be one of the postulates the equivalence of which will be verified: $n \in N$.

(1) $\sum_{i \in \boldsymbol{N}_{n}} \beta_{i} P_{i}=0 \Leftrightarrow \sum_{i \in \boldsymbol{N}_{n}} \beta_{i} V_{P_{2}}=0$ with $\beta_{i} \in \boldsymbol{R}$ for every $i \in \boldsymbol{N}_{n}$ and any

(2) $\left\langle V_{P}, Q\right\rangle=\left\langle V_{Q}, P\right\rangle$ for all $P, Q \in A(G)$.

Theorem 3. If the postulate (1) holds, then the bijection $\underline{J}: A(G) \rightarrow A(W)$ defined by $\underline{J}(P)=\left\{V_{P}\right\}$ for every $P \in A(G)$ has a unique extension $J: B^{\prime} \rightarrow B$ which is

(i) linear and bijective, (ii) canonical, (iii) order preserving in both directions.

Proof. (i) Given any basis $S=\left\{P_{1}, \ldots, P_{N}\right\} \subset A(G)$ of $B^{\prime}$, then, by (1), $\sum_{i \in \boldsymbol{N}_{N}} \beta_{i} V_{\boldsymbol{P}_{\mathrm{t}}}=0$ implies $\sum_{i \in \boldsymbol{N}_{N}} \beta_{i} P_{i}=0$, hence $\beta_{i}=0$ for all $i \in \boldsymbol{N}_{N}$ and thus $\underline{J}[S]$ is a basis of $B$. Consequently, there exists a unique linear extension $J_{S}: B^{\prime} \rightarrow B$ which is-by bijectivity of $\underline{J}$ and $\operatorname{lin}_{\boldsymbol{R}} S=B^{\prime}$-bijective, too.

(ii) Let $T=\left\{Q_{1}, \ldots, Q_{N}\right\} \subset A(G)$ be another basis of $B^{\prime}$ and $J_{T}$ the corresponding linear bijective extension of $\underline{J} \mid T$ to $B^{\prime}$. Then any $R \in A(G)$ has the representations

$$
R=\sum_{i \in \boldsymbol{N}_{N}} \beta_{i} P_{i} \text { and } R=\sum_{i \in \boldsymbol{N}_{N}} \beta_{i}^{\prime} Q_{i} \text { with } \beta_{i}, \beta_{i}^{\prime} \in \boldsymbol{R} \text { for all } i \in \boldsymbol{N}_{N} .
$$

Utilizing (1) again, we conclude from $\sum_{i \in \boldsymbol{N}_{N}} \beta_{i} P_{i}-\sum_{i \in \boldsymbol{N}_{N}} \beta_{i}^{\prime} Q_{i}=0$ that $\sum_{i \in \boldsymbol{N}_{N}} \beta_{i} V_{P_{i}}=\sum_{i \in \boldsymbol{N}_{N}} \beta_{i}^{\prime} V_{Q_{i}}$. So, $J_{S}(R)=\sum_{i \in \boldsymbol{N}_{N}} \beta_{i} J\left(P_{i}\right)=\sum_{i \in \boldsymbol{N}_{N}} \beta_{i} V_{P_{i}}$ and $J_{T}(R)$ $=\sum_{i \in N_{N}} \beta_{i}^{\prime} \underline{J}\left(Q_{i}\right)=\sum_{i \in N_{N}} \beta_{i}^{\prime} V_{Q_{i}}$, hence $J_{S}(R)=J_{T}(R)$ for all $R \in A(G)$. Therefore, $J_{S}=: J$ is independent of bases consisting only of atoms. $J$ is even independent of any basis of $B^{\prime}$ because $A(G)$ generates $B^{\prime} . J^{-1}$ is also canonical because $J$ is so. 
(iii) For every atom $Q \in A(G)(1)$ implies $Q=\sum_{i \in N_{N}} \beta_{i} P_{i} \Leftrightarrow V_{Q}=\sum_{i \in N_{N}} \beta_{i} V_{P_{\mathrm{\imath}}}$, whence the desired restrictions $J \mid A(G)=\underline{J}$ and $J^{-1} \mid A(W)=\underline{J}^{-1}$ follow. There remains only to show that $J\left[B_{+}^{\prime}\right]=B_{+}$and $J^{-1}\left[B_{+}\right]=B_{+}^{\prime}$ : every $Y \in B_{+}^{\prime}$ has a representation (cf. [5]) by $Y=\sum_{i \in N_{n}} \beta_{i} P_{i}, \beta_{i} \in R_{+}^{*}$ for all $i \in \boldsymbol{N}_{n}$. Applying $J$ to $Y$, we get $J(Y)=\sum_{i \in \boldsymbol{N}_{n}} \beta_{i} J\left(P_{i}\right)=\sum_{i \in \boldsymbol{N}_{n}} \beta_{i} V_{P_{\imath}} \in B_{+}$ and, moreover, every $X \in B_{+}$is the image of an $Y \in B_{+}^{\prime}$ under $J$. Conversely, if $X \in B_{+}$, then $X=\sum_{j \in N_{m}} \beta_{j}^{\prime} V_{Q_{j}}, \beta_{j}^{\prime} \in \boldsymbol{R}_{+}^{*}$ for all $j \in \boldsymbol{N}_{m}$. Again by (1), $J^{-1}(X)=\sum_{j \in \boldsymbol{N}_{m}} \beta_{j}^{\prime} J^{-1}\left(V_{Q_{j}}\right)=\sum_{j \in \boldsymbol{N}_{m}} \beta_{j}^{\prime} Q_{j} \in B_{+}^{\prime}$ is obtained. This completes the proof.

Theorem 4. With the hypothesis of Theorem 3 there holds

(i) $\left\langle V_{P}, Q\right\rangle=\left\langle V_{Q}, P\right\rangle$ for all $P, Q \in A(G)$ (postulate (2))

(ii) $J$ is symmetrical ( $J^{t}$ denoting the transposed isomorphism of $J$ ).

Proof. (i) $J^{t}$ is defined by $\langle J \bar{Y}, Y\rangle=\left\langle\bar{Y}, J^{t} Y\right\rangle$, i.e. by $\mu(J \bar{Y}, Y)$ $=\mu\left(J^{t} Y, \bar{Y}\right)$ for all $\bar{Y}, Y \in B^{\prime} . B^{\prime}, B$ being finite-dimensional, $J^{t}: B^{\prime} \rightarrow B^{\prime \prime}=B$ is valid. For any fixed $Q \in A(G)$ and every $P \in A(G)$ we obtain $\left\langle V_{P}, Q\right\rangle$ $=\langle J P, Q\rangle=\left\langle P, J^{t} Q\right\rangle$ and thus $0 \leqq\left\langle P, J^{t} Q\right\rangle \leqq 1$ for every $P \in A(G)$. So, $J^{t} Q=: X^{Q} \in B_{+}$, whence $X^{Q}=\lambda^{Q} V^{Q}$ with $V^{Q} \in K$ and $\lambda^{Q} \in \boldsymbol{R}_{+}^{*}$. $X^{Q}=0$, indeed, is excluded by $J^{t}$ being an isomorphism. Next we shall show $\lambda^{Q}=1$ and so, as a consequence, $V^{Q}=V_{Q}$. To this purpose let us consider any orthodecomposition of $\mathbf{1}$ such that $\mathbf{1}=Q+\sum_{i \in \boldsymbol{N}_{n}} P_{i}$ and $P_{i} \in A(G)$. Utilizing (1), we conclude that $\langle J 1, Q\rangle=\left\langle V_{Q}, Q\right\rangle+\sum_{i \in N_{n}}\left\langle V_{P_{i}}, Q\right\rangle$ holds, hence $\langle J \mathbf{1}, Q\rangle=\left\langle V_{Q}, Q\right\rangle=1$. Thus $\left.1=\langle J \mathbf{1}, Q\rangle=\left\langle\mathbf{1}, J^{t} Q\right\rangle\right\rangle$ $=\left\langle\mathbf{1}, X^{Q}\right\rangle=\lambda^{Q}\left\langle\mathbf{1}, V^{Q}\right\rangle=\lambda^{Q}$. Finally, substituting $Q$ for $P$ in the equation $\left\langle V_{P}, Q\right\rangle=\left\langle P, J^{t} Q\right\rangle=\left\langle P, V^{Q}\right\rangle$ yields $\left\langle Q, V^{Q}\right\rangle=1$. Since $K_{1}(Q)$ is the singleton $\left\{V_{Q}\right\}$, so $V^{Q}=V_{Q}$. This proves (2).

(ii) As a consequence of $J\left|A(G)=J^{t}\right| A(G)$ from (2) we even get $J=J^{t}$ because $A(G)$ generates $B^{\prime}$.

Theorem 5. Postulate (2) implies postulate (1).

Proof. Suppose that any finite $S:=\left\{P_{1}, \ldots, P_{n}\right\} \subset A(G)$ satisfies $\sum_{i \in N_{n}} \beta_{i} P_{i}=0$ with $\beta_{i} \in \boldsymbol{R}$ for all $i \in N_{n}$. Then for every $Q \in A(G)$, there holds $\left\langle V_{Q}, \sum_{i \in N_{n}} \beta_{i} P_{i}\right\rangle=\sum_{i \in N_{n}} \beta_{i}\left\langle V_{Q}, P_{i}\right\rangle=0$. An application of (2) yields $0=\sum_{i \in \boldsymbol{N}_{n}} \beta_{i}\left\langle V_{Q}, P_{i}\right\rangle=\sum_{i \in \boldsymbol{N}_{n}} \beta_{i}\left\langle V_{P_{i}}, Q\right\rangle=\left\langle\sum_{i \in \boldsymbol{N}_{n}} \beta_{i} V_{\boldsymbol{P}_{i}}, Q\right\rangle$. Hence $\sum_{i \in \boldsymbol{N}_{n}} \beta_{i} V_{P_{l}}=0$, which completes the proof.

Corollary. The postulates (1) and (2) are equivalent. 


\section{II. $B^{\prime}$ as a Hilbert-Space}

The most important result from (2) is that $B^{\prime}$ (and $B$ as well) becomes a real Hilbert space.

Theorem 6. If (2) is valid and $J$ denotes the isomorphism from Theorem 3, then the bilinear functional $\langle\cdot \mid \cdot\rangle: B^{\prime} \times B^{\prime} \rightarrow \boldsymbol{R}$ defined by $\langle\bar{Y} \mid Y\rangle:=\langle J \bar{Y}, Y\rangle=\mu(J \bar{Y}, Y)$ for all $\bar{Y}, Y \in B^{\prime}$ is an inner product on $B^{\prime}$.

Proof. (i) Bilinearity of $\langle\cdot \mid \cdot\rangle$ is obvious.

(ii) To prove strict positivity of $\langle\cdot \mid \cdot\rangle$ we remember that every $Y \in B^{\prime}$ has a spectral representation (cf. [5]) by $Y=\sum_{i \in N_{n}} \beta_{i} P_{i}, \beta_{i} \in R, P_{i} \in A(G)$ for all $i \in N_{n}, P_{i} \perp P_{k}$ for all $i, k \in N_{n}$ and $i \neq k$. Therefore, $\langle Y \mid Y\rangle=\langle J Y, Y\rangle$ $=\sum_{i, k \in N_{n}} \beta_{i} \beta_{k}\left\langle V_{P_{\imath}}, P_{k}\right\rangle=\sum_{i \in N_{n}} \beta_{i}^{2} \geqq 0$. Hence $\langle Y \mid Y\rangle=0$ iff $Y=0$.

(iii) Symmetry of $\langle\cdot \mid \cdot\rangle$ follows from symmetry of $J$ : for all $\bar{Y}, Y \in B^{\prime}$ there holds $\langle\bar{Y} \mid Y\rangle=\langle J \bar{Y}, Y\rangle=\left\langle\bar{Y}, J^{t} Y\right\rangle=\langle\bar{Y}, J Y\rangle=\mu(J Y, \bar{Y})=\langle Y \mid \bar{Y}\rangle$.

Theorem 7. $\left(B^{\prime},\langle\cdot \mid \cdot\rangle\right)$ is a real Hilbert space.

Proof. Since $B^{\prime}$ is a real finite-dimensional Banach space with respect to the supremum norm and since the norm induced by $\langle\cdot \mid \cdot\rangle$ on $B^{\prime}$ is equivalent with that one, $B^{\prime}$ is also complete with respect to the inner product norm. Thus $B^{\prime}$ is a real Hilbert space and, being self-dual, it coincides with $B$.

Theorem 8. The lattice-theoretical orthogonality relation on $G$ equals that which is induced on $G$ by the inner product $\langle\cdot \mid \cdot\rangle$ of Theorem 6.

Proof. All $E_{1}, E_{2} \in G$ have lattice-theoretically atomic orthodecompositions by $E_{1}=\sum_{i \in \boldsymbol{N}_{n}} P_{i}$ and $E_{2}=\sum_{j \in \boldsymbol{N}_{m}} Q_{j}$.

(i) Suppose that $\left\langle E_{1} \mid E_{2}\right\rangle=0$. Then $\sum_{\substack{i \in \boldsymbol{N}_{n} \\ j \in \boldsymbol{N}_{m}}}\left\langle P_{i} \mid Q_{j}\right\rangle=0$ and so, by positivity of each $\left\langle P_{i} \mid Q_{j}\right\rangle$, there must hold $P_{i} \in K_{0}\left(Q_{j}\right)=K_{1}\left(Q_{j}^{\perp}\right)$ for all $i \in N_{n}$ and all $j \in N_{m}$. Hence $P_{i} \leqq Q_{j}^{\perp}$, whence we infer $E_{1}=\sum_{i \in N_{n}} P_{i}$ $\leqq \bigwedge_{j \in \boldsymbol{N}_{m}} Q_{j}^{\perp}=\left\lceil\sum_{j \in \boldsymbol{N}_{m}} Q_{j}\right]^{\perp}=E_{2}^{\perp}$

(ii) The converse follows from reading (i) in the reverse direction.

Another consequence of the inner product on $B^{\prime}$ is the existence of an ortho-additive dimension function on $G$.

Theorem 9. (i) The function $d: G \rightarrow \boldsymbol{R}_{+}$defined by $d(E)=\langle\mathbf{1} \mid E\rangle$ for all $E \in G$ is isotone on $G$. 
(ii) Im $d \subset N \cup\{0\}$. (iii) For any $\in E_{1}, E_{2} \in G$ : if $E_{1}<E_{2}$, then all maximal orthochains connecting $E_{1}$ to $E_{2}$ possess the same length.

Proof. By Theorem 8, there holds $d(E)=\left\langle E+E^{\perp} \mid E\right\rangle=\langle E \mid E\rangle$ for all $E \in G$. Choose any orthodecomposition $\sum_{i \in N_{n}} P_{i}$ of $E$, then $d(E)=\sum_{i, j \in \boldsymbol{N}_{n}}\left\langle P_{i} \mid P_{j}\right\rangle=\sum_{i, j \in \boldsymbol{N}_{n}} \delta_{i j}=n$. Therefore, $d(E)$ counts the atoms in the above atomic orthodecomposition. By the function property of $d$, any orthodecomposition of $E$ different from the above must have the same number of atoms, namely $d(E)=n$, which proves (ii) and (iii). Utilizing atomicy and orthomodularity of $G$ implies isotony of $d$, which proves (i).

$d$ being normalized by $\frac{d(E)}{d(\mathbf{1})}=: \delta(E)$ for all $E \in G$, the range of the function $\delta$ satisfies $\{0,1\} \subseteq \operatorname{Im} \delta \subset[0,1] \cap Q$. To state the defining relations for $\delta$ to be an ortho-additive dimension function on $G$, observe that immediately from the definitions of $d$ and $\delta$ there results

(i) $\delta(0)=0, \delta(\mathbf{1})=1 ; \delta\left(E_{1} \vee E_{2}\right)=\delta\left(E_{1}\right)+\delta\left(E_{2}\right)$ for all $E_{1}, E_{2} \in \mathrm{G}$ such that $E_{1} \perp E_{2}$ (finite ortho-additivity of $\delta$ ). This fact is summarized in the

Theorem 10. The normalized, $Q$-valued function $\delta$ on $G$ defined by $\delta(E):=\frac{d(E)}{d(\mathbf{1})}$ for all $E \in G$ is an ortho-additive dimension function on $G$.

$\delta$ permits us to introduce a dimensional equivalence relation $\sim$ on $G$ by:

For all $E_{1}, E_{2} \in G: E_{1} \sim E_{2}$ iff $\delta\left(E_{1}\right)=\delta\left(E_{2}\right)$ (cf. [4]). This equivalence relation is connected to the lattice operations as follows:

(A) $E \sim 0$ implies $E=0$.

(B) $E \sim E_{1} \vee E_{2}$ implies the existence of $E_{1}^{\prime}, E_{2}^{\prime} \in G$ such that $E=E_{1}^{\prime} \vee E_{2}^{\prime}$ and $E_{i}^{\prime} \sim E_{i}$ for all $i \in N_{2}$.

(C) Let $\left(E_{i}^{\prime}\right)_{i \in \boldsymbol{N}_{n}}$ and $\left(E_{i}^{\prime \prime}\right)_{i \in \boldsymbol{N}_{n}}$ be (finite) sequences of pairwise orthogonal elements of $G$ ("finite" because of $\operatorname{dim} B^{\prime}=N<\infty$ ). If $E_{i}^{\prime} \sim E_{i}^{\prime \prime}$ for every $i \in N_{n}$, then $\bigvee_{i \in N_{n}} E_{i}^{\prime} \sim \bigvee_{i \in N_{n}} E_{i}^{\prime \prime}$.

(D) If $E_{1} \not E_{2}$, then there exist $E_{i}^{\prime} \neq 0$ such that $E_{i}^{\prime} \leqq E_{i}$ for all $i \in N_{2}$ and $E_{1}^{\prime} \sim E_{2}^{\prime}$.

(A)-(D) result directly from $G$ being orthomodular and atomic. In the sense of Loomis [4] $G$ is a dimension lattice, whereas other authors prefer to postulate: 
(D') Perspective elements of $G$ are equivalent instead of (D) in order to call $G$ a dimension lattice. Loomis [4] proves $\left(D^{\prime}\right) \Rightarrow(D)$. The validity of the converse is, in general, unknown. However, in the special case of $G$ treated here we shall verify modularity of $G$ and thus $(D) \Rightarrow\left(D^{\prime}\right)$ by first showing the validity of the Jordan-Dedekind chain condition and then the so-called "covering condition" for $G$ (cf. [6]).

Theorem 11. For all $\bar{E}, E \in G$ : if $\bar{E} \leqq E$, then all covering chains connecting $\bar{E}$ and $E$ possess the same length.

Proof. Suppose $\bar{E}<E . \operatorname{dim} B^{\prime}=N<\infty$, atomicy and orthomodularity guarantee the existence of covering chains connecting $\bar{E}$ and $E$. Let $\bar{E} \lessdot \bar{E} \vee P_{1} \lessdot \bar{E} \vee P_{1} \vee P_{2} \lessdot \cdots \lessdot \bar{E} \vee \bigvee_{i \in N_{n}} P_{i}=E$ be any covering chain between $\bar{E}$ and $E$ with $P_{i} \in A(G)$ for all $i \in N_{n}$. Defining $E_{j}:=\bar{E} \vee \bigvee_{i \in N_{j}} P_{i}$ for every $j \in N_{n}$, we obtain $E_{n}=E=E_{n-1} \vee\left(E \wedge E_{n-1}^{\perp}\right)$ by orthomodularity of $G$ and, since $E$ covers $E_{n-1}, Q_{1}:=E \wedge E_{n-1}^{\perp} \in A(G)$. Similarly, since $E_{n-1}$ covers $E_{n-2}$, so $Q_{2}:=E_{n-1} \wedge E_{n-2}^{\perp} A(G)$. Continuing this procedure, we get a covering chain between $\bar{E}$ and $E$ which is constructed by $n$ pairwise orthogonal atoms. Hence $d(E)-d(\bar{E})=n$. Considering any covering chain between $\bar{E}$ and $E$ different from the above and having the length $m$, then, to this one there corresponds a covering chain constructed by $m$ pairwise orthogonal atoms via the same arguments as above. Then Theorem 9 requires $m=n$.

Corollary. For all $P, Q \in A(G)$ : if $P \perp Q$, then $G(0, P \forall Q)$ is a modular sublattice of $G$.

Proof. For all $E^{\prime}, E^{\prime \prime} \in G(0, P \vee Q) d$ satisfies the dimension equation $d\left(E^{\prime}\right)+d\left(E^{\prime \prime}\right)=d\left(E^{\prime} \vee E^{\prime \prime}\right)+d\left(E^{\prime} \wedge E^{\prime \prime}\right)$.

Moreover, there holds the

Theorem 12. For all $P, Q \in A(G): G(0, P \vee Q)$ is a modular sublattice of $G$.

Proof. By the preceding corollary only the case of $P \not Q$ needs to be investigated. $d(P \vee Q)=2$ must be shown: orthomodularity of $G$ implies $P \vee Q=P \vee\left((P \vee Q) \wedge P^{\perp}\right)=P+\left((P \vee Q) \wedge P^{\perp}\right) \quad$ and $\quad P \vee Q=Q$ $+\left((P \vee Q) \wedge Q^{\perp}\right), \quad$ whence $\quad \frac{1}{2}(P \vee Q)=\frac{1}{2} P+\frac{1}{2}(P \vee Q) \wedge P^{\perp}=\frac{1}{2} Q$ $+\frac{1}{2}(P \vee Q) \wedge Q^{\perp}$. This implies that the line segments $\left[P,(P \vee Q) \wedge P^{\perp}\right]$ and $\left[Q,(P \vee Q) \wedge Q^{\perp}\right]$ intersect in $\frac{1}{2}(P \vee Q)$. Thus they span a plane in $B^{\prime}(P \vee Q)$. Take $\frac{1}{2}(P+Q)$ in this plane and consider the line segment $\left[\frac{1}{2}(P+Q), \frac{1}{2}(P \vee Q)\right]$ which is, because of $P \not Q$, not a singleton. To exclude $\frac{1}{2}(P+Q) \in \partial L_{P \vee Q}$, assume the contrary. Then the extremal hull of $\left\{\frac{1}{2}(P+Q)\right\}$, which contains $K_{1}(P \vee Q)=C\left(\frac{1}{2} P+\frac{1}{2} Q\right)$, is contained in $\partial L_{P \vee Q}$. Since $\operatorname{lin}_{R} K_{1}(P \vee Q)=B(P \vee Q)$, we obtain $B(P \vee Q) \subset \operatorname{lin}_{R} L_{P \vee Q}$ 
$=B^{\prime}(P \vee Q)$. This proper inclusion contradicts the isomorphy of $B(P \vee Q)$ and $B^{\prime}(P \vee Q)$. Thus $\frac{1}{2}(P+Q)$ is an internal point of $L_{P \vee Q}$ and therefore the extension of the line segment $\left[\frac{1}{2}(P+Q), \frac{1}{2}(P \vee Q)\right]$ via $\frac{1}{2}(P+Q)$ intersects $\partial L_{P \vee Q}$ in a point $F$. By construction we have $\frac{1}{2}(P+Q)=\lambda F$ $+(1-\lambda) \frac{1}{2}(P \vee Q)$ with $\left.\lambda \in\right] 0,1[. F$ being an effect, it is a convex combination $F=\sum_{i \in \boldsymbol{N}_{m}} \lambda_{i} E_{i}$ of $m$ extreme points $E_{i} \in G(0, P \vee Q)$ of $L_{P \vee Q}$. Hence we conclude that $\langle F \mid P \vee Q\rangle=\sum_{i \in N_{m}} \lambda_{i}\left\langle E_{i} \mid P \vee Q\right\rangle \geqq 1$. Since $\left\langle\frac{1}{2}(P+Q) \mid P \vee Q\right\rangle=1$, there holds $1=\left\langle\frac{1}{2}(P+Q) \mid P \vee Q\right\rangle=\lambda\langle F \mid P \vee Q\rangle$ $+(1-\lambda) \frac{1}{2}\langle P \vee Q \mid P \vee Q\rangle=\lambda\langle F \mid P \vee Q\rangle+(1-\lambda) \frac{1}{2} d(P \vee Q)$ whence we infer $\langle F \mid P \vee Q\rangle=1$ and $d(P \vee Q)=2 . d(P \vee Q)=2$ is equivalent with modularity of $G(0, P \vee Q)$.

As the final step towards modularity of $G$ we shall verify the validity of the covering condition for $G$ :

Theorem 13. For all $E_{1}, E_{2} \in G$ : if $E_{1}, E_{2}$ both cover $E_{1} \wedge E_{2}$, then $E_{1} \vee E_{2}$ covers $E_{1}$ and $E_{2}$.

Proof. By hypothesis there exist two atoms $P, Q \in A(G)$ such that $E_{1}=\left(E_{1} \wedge E_{2}\right) \vee P$ and $E_{2}=\left(E_{1} \wedge E_{2}\right) \vee Q$. So we obtain $\left(E_{1} \wedge E_{2}\right)$ $\vee(P \vee Q)=E_{1} \vee E_{2}=E_{1} \vee Q=E_{2} \vee P$. Consequently, applying $d$ to these equations and utilizing Theorem 12 , we have $d\left(E_{1}\right)=d\left(E_{1} \wedge E_{2}\right)$ $+d(P)=d\left(E_{1} \wedge E_{2}\right)+1=d\left(E_{1} \wedge E_{2}\right)+d(Q)=d\left(E_{2}\right)$ and $d\left(E_{1} \vee E_{2}\right)$ $=d\left(E_{1} \wedge E_{2}\right)+d(P \vee Q)=d\left(E_{1} \wedge E_{2}\right)+2$. Hence we infer $d\left(E_{1} \vee E_{2}\right)$ $=d\left(E_{1}\right)+1=d\left(E_{2}\right)+1$ which expresses that $E_{1} \vee E_{2}$ covers $E_{1}$ and $E_{2}$.

Now we can formulate the main result of this paper in the

Theorem 14. If $B^{\prime}$ is a Hilbert space equipped with the inner product from Theorem 6, then the orthomodular lattice $G$ is even modular.

Proof. With the hypothesis of Theorem 12 MacLaren has proved the assertion in [6].

Corollary 1. $d$ and $\delta$ are dimension functions on $G$.

Corollary 2. (D) implies (D').

Remark 3. The symmetry postulate (2) implies modularity of $G$. Thus modularity of $G$ is the consequence of certain possibilities of constructing preparing and effect portions of technical apparatuses for physical experiments. To be precise: $V_{P}, V_{Q}$ are irreducible ensembles causing the atomic decision effects $P$ and $Q$, respectively, with probability $1 . V_{P}$ produces the atomic decision effect $Q$ with probability $\left\langle V_{P}, Q\right\rangle$. Then the symmetry postulate (2) says that the effect apparatuses characterized by $P$ react to the ensemble $V_{Q}$ with same probability as $Q$ 
reacts to $V_{P}$. This fact is well-known in ordinary quantum mechanics based on the Hilbert space model where $\left\langle V_{P}, Q\right\rangle$ is given by $\left\langle V_{P}, Q\right\rangle$ $=\operatorname{Tr}(P Q)$.

This promises another deduction of the Hilbert space model of quantum mechanics than that one given by Stolz [7] who used the coordinatizing procedure of projective geometry and, therefore, must necessarily involve the additional dimension postulate of $d[G] \geqq 4$ in his exposition.

We conclude this section by showing that the involution on $\mathscr{B}\left(B^{\prime}\right)$ induced by the Hilbert space property of $B^{\prime}$ has $\mathscr{T}(G)$ in its set of fixed elements. This question was broached in [2]. There we proved that the algebra $\mathscr{B}\left(B^{\prime}\right)$ is generated by the physical filters $\boldsymbol{T}_{E}$ provided $G$ is irreducible. By the Hilbert space structure on $B^{\prime}$ induced by the symmetry postulate $(2) \mathscr{B}\left(B^{\prime}\right)$ becomes a $B^{*}$-algebra and its generators $\boldsymbol{T}_{E}$ are selfadjoint projectors on $B^{\prime}$.

Theorem 15. Concerning the involution * on $\mathscr{B}\left(B^{\prime}\right)$ induced by the inner product $\langle\cdot \mid \cdot\rangle$ on $B^{\prime}$ there holds $\boldsymbol{T}_{E}^{*}=\boldsymbol{T}_{E}$ for all $E \in G$.

Proof. Remember that $\boldsymbol{T}_{E}$ was generally defined by $\left\langle V, \boldsymbol{T}_{E} F\right\rangle=\langle V, F\rangle$ for all $V \in K_{1}(E)$ and every $F \in L$. Self-adjointness of any idempotent $\boldsymbol{T}_{E} \in \mathscr{T}(G)$ is equivalent with $\boldsymbol{T}_{E}$ being a perpendicular projector on $B^{\prime}$ (e.g. [3]). That is we have only to verify $\operatorname{Ker} \boldsymbol{T}_{E}=B^{\prime}(E)^{\perp}$ for all $E \in G$ concerning the orthogonal sum $B^{\prime}=B^{\prime}(E) \oplus B^{\prime}(E)^{\perp}$ :

(i) For every $Y \in B^{\prime}(E)^{\perp}$ we have $\langle P \mid Y\rangle=0$ for all $P \in L_{E}$ i.e. for all $P \leqq E$. Then from $\left\langle P \mid \boldsymbol{T}_{E} Y\right\rangle=\langle P \mid Y\rangle=0$ for all $P \in K_{1}(E) \subset L_{E}$ there follows $\boldsymbol{T}_{E} Y \in B^{\prime}(E)^{\perp} \cap B^{\prime}(E)=\{0\}$, hence $Y \in \operatorname{Ker} \boldsymbol{T}_{E}$ and thus $B^{\prime}(E)^{\perp}$ $\subseteq \operatorname{Ker} \boldsymbol{T}_{E}$.

(ii) For every $Y \in \operatorname{Ker} \boldsymbol{T}_{E}$ there holds $\left\langle P \mid \boldsymbol{T}_{E} Y\right\rangle=\langle P \mid Y\rangle=0$ for all $P \in K_{1}(E)$ i.e. for all $P \leqq E$. Therefore $Y \in B^{\prime}(E)^{\perp}$ because $K_{1}(E)$ generates $B^{\prime}(E)$; consequently $\operatorname{Ker} \boldsymbol{T}_{E} \cong B^{\prime}(E)^{\perp}$.

There remain three open questions:

1. Does modularity of $G$ imply the symmetry postulate (2) without the requirement of $d(G) \geqq 4$ ?

2. Suppose that the filter algebra $\mathscr{B}\left(B^{\prime}\right)$ possesses an involution * such that $\boldsymbol{T}^{*} \boldsymbol{T}=\mathbf{0}$ implies $\boldsymbol{T}=\mathbf{0}$ and such that $\boldsymbol{T}_{E}^{*}=\boldsymbol{T}_{E}$. Does then the symmetry postulate (2) hold?

3. It is possible to deduce the Hilbert space model of quantum mechanics from the symmetry postulate (2) without any use of projective geometry? 


\section{References}

1. Dähn,G.: Attempt of an axiomatic foundation of quantum mechanics and more general theories. IV. Commun. math. Phys. 9, 192-211 (1968).

2. Dähn, G. : The algebra generated by physical filters. Submitted to Commun. math. Phys.

3. Halmos, P. R.: Finite-dimensional vector spaces. $2^{\text {nd }}$. Princeton: Van Nostrand 1958.

4. Loomis, L.H.: The lattice-theoretic background of the dimension theory of operator algebras. Mem. Am. Math. Soc. 18, 1-36 (1955).

5. Ludwig, G.: Attempt of an axiomatic foundation of quantum mechanics and more general theories. III. Commun. math. Phys. 9, 1-12 (1968).

6. MacLaren, M.D.: Atomic orthocomplemented lattices. Pacific J. Math. 14, 597-612 (1964).

7. Stolz, P.: Attempt of an axiomatic foundation of quantum mechanics and more general theories. V. Commun. math. Phys. 11, 303-313 (1969).

Günter Dähn

Mathematisches Institut der Universität

D-7400 Tübingen

Brunnenstraße 27

Federal Republic of Germany 